\title{
The ERES Digital Library: Online Access to Proceedings
}

\author{
Bob Martens \\ Vienna University of Technology, A-1040 Vienna, Austria \\ e-mail: martens@cec.tuwien.ac.at
}

Keywords: Electronic Publishing, Archival, Open Access, Topics of Real Estate

\begin{abstract}
This contribution presents the initial steps in the creation of a Digital Library consisting of previous ERES proceedings. The series of ERES conferences started in 1993, but their visibility has predominantly been limited to the ERES website. Sixteen conferences related to Real Estate resulted in an interesting output of over two thousands papers and it makes sense to offer them to a broader audience in an easily accessible way. Nowadays, average "annual paper production" amounts to approx. 200 entries and the use of this growing amount of information requires guidance. This includes the option to search for entries of a certain author (within a certain time span), as well a possibility to retrieve records on a specific topic related to Real Estate. First results on the gathering of initial content will be presented. At the time of writing over 1,500 entries are recorded. In the framework of this contribution, ideas on future developments related to the ERES Digital Library will also be presented.
\end{abstract}

\section{$1 \quad$ Introduction}

The European Real Estate Society has a long-standing Annual Conference tradition. Since 1993, hundreds of papers have been prepared and presented at the meetings. However, a local conference organisation is mainly interested in publishing the proceedings of the year they are in charge of. The need for archiving goes beyond a certain year and would support the possibility to access a kind of collective memory. In other words, both presenters and (future) audiences would be able to trace what was happening at any given point in time in a certain real-estate related area.

Ideally, this would include not only abstracts and full papers, but also the presentation sheets (e.g. Powerpoint) and, preferably, the references and literature citations in a separate database on the Digital Library portal. Sometimes it is assumed, that the online availability of materials would lead to a reduced audience. 
Though this is hard to verify, some delay can be built in and the level of available chunks of information can be controlled when recoding entries in a Digital Library.

\section{Setup of the Digital Library}

As a non-profit organisation, the ERES-Society is not driven by plainly commercial motives. The creation and setup of the ERES Digital Library also abides by this principle and leaves the revenues in the hands of the society itself. Moreover, a higher degree of visibility and possible enhancement of impact would support prosperous development. Enormous progress has been made in the area of webbased technologies. The user interface of the ERES Digital Library (eres.scix.net) was created in the framework of an EU-IST project called SciX ("Open Scientific Exchange of Information") [1]. The necessity of handling and maintaining it on a shoestring budget must be borne in mind. Another crucial point is the initial filling of the library with (rich) content.

\subsection{Gathering and Recording of Data}

It does not surprise that the acquisition of material stemming from recent conferences is easier to manage than that from the early days. The persons in charge may have changed and/or shifted their interests. Furthermore, activities usually cease once the conference is settled and the proceedings are printed. A couple of years ago proceedings started to be published on CD-Rom, but all these materials are likely to be stored in the cupboards and computers of individual attendees (with limited "access").

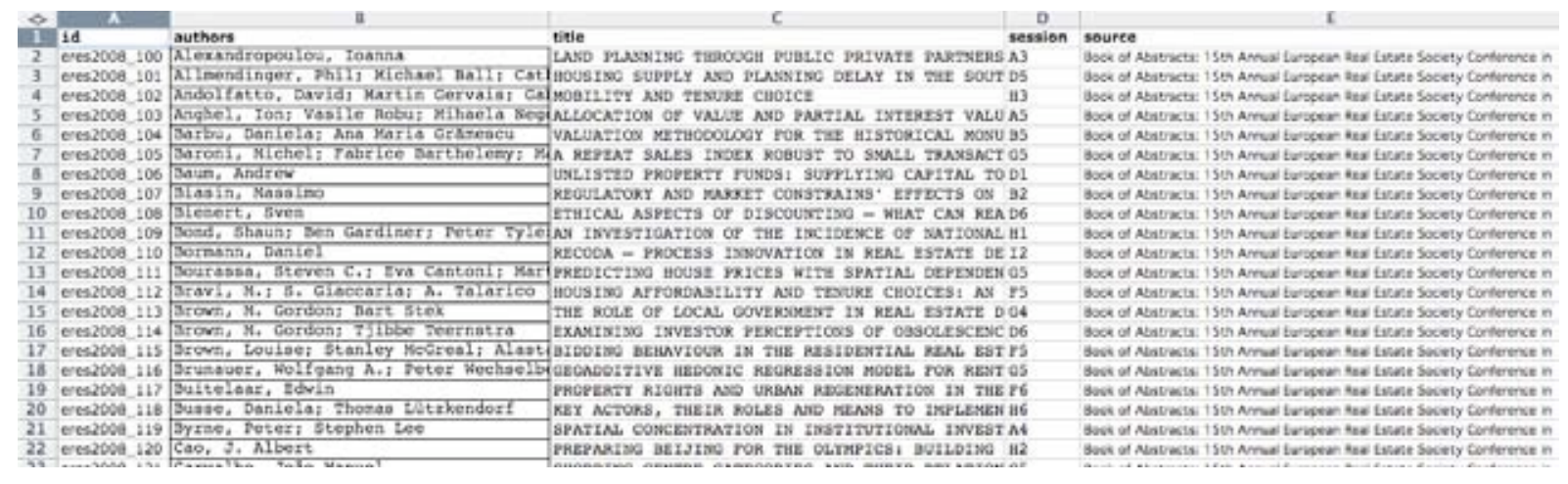

Fig. 1 Example of extracted metadata (to be imported at eres.scix.net)

It seemed relatively easy to deal with the time-span from 2003 onward. Grateful mention must be made of the support provided by previous conference chairs in the form of making the data available, as well as their support by extracting bits and 
pieces which had to be consolidated in a chart with metadata (Fig. 1). The collected sets of structured metadata allowed for an import into the ERES Digital Library. Ideally, not only authors and titles of the presentation are to be recorded, but also the abstracts and the sessions, in which the papers were presented. The largest number of papers stems from the Annual Conference itself (approx. 1,500 items), but also the contributions to the "Education Seminar" (with nearly 100 entries) were recorded.

\begin{tabular}{|c|c|c|}
\hline \multicolumn{3}{|c|}{ Search Results } \\
\hline \multicolumn{3}{|c|}{ Search for Taltandil } \\
\hline \multicolumn{3}{|c|}{ 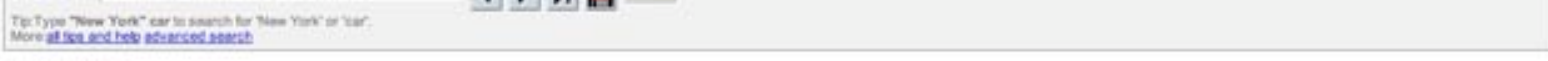 } \\
\hline \multicolumn{3}{|c|}{ Hets it to 20 of 21} \\
\hline - detals ful & citation & $\begin{array}{l}\text { check } \\
\text { to } \\
\text { select }\end{array}$ \\
\hline 1. & 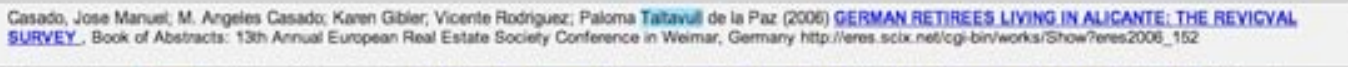 & B \\
\hline 2. 칠 & 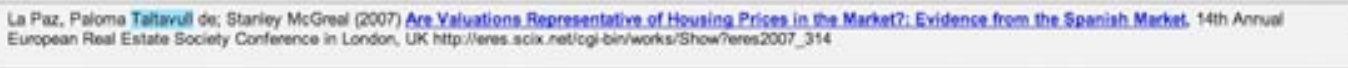 & 日 \\
\hline 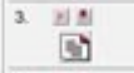 & 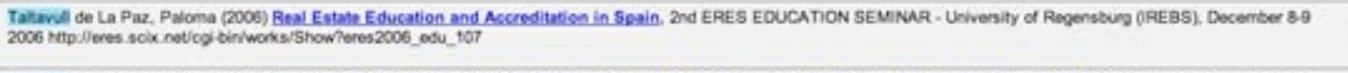 & B \\
\hline $\begin{array}{l}\text { 4. 처 } \\
\text { [5] }\end{array}$ & 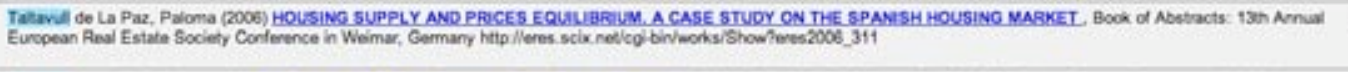 & B \\
\hline 5. 20 & 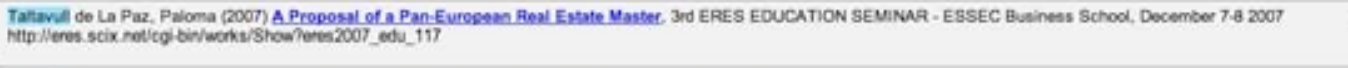 & Q \\
\hline 6. 20 & 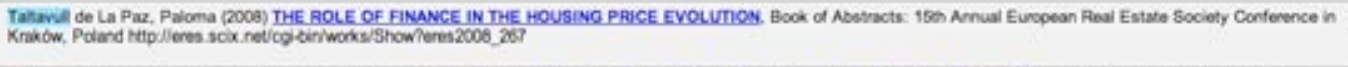 & 早 \\
\hline 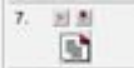 & 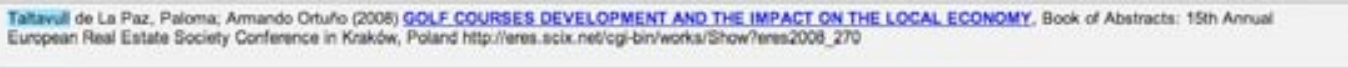 & B \\
\hline
\end{tabular}

Fig. 2 Recorded entries, displaying the results of a search for "Taltavull"

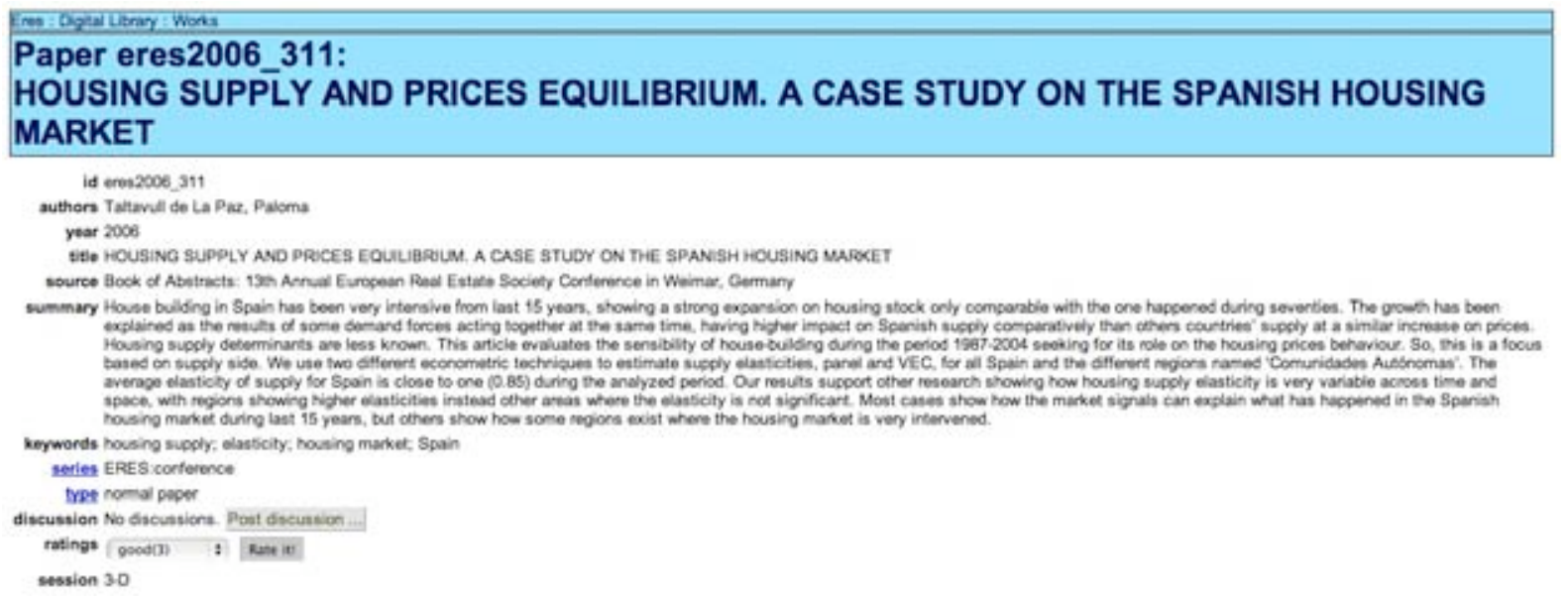

Fig. 3 Example of the recorded information for an individual entry 


\subsection{Developmental Steps}

It has to be noted that there is currently a body of initial content operational at eres.scix.net. Extending it with further entries from forthcoming conferences should have a high priority, as these materials are the most recent ones and therefore of utmost interest. In a next stage, the addition of the corresponding full papers could be considered.

The question as to whether it makes sense to record missing entries from the period 1993-2002 is hard to answer. It would result in another 1,000 entries. Not all the material may still be available in a digital format and digitalization may be required. However, the investment would make sense in order to provide an overview of progress made in the field since 1993 [2]. Again, with the support of previous conference organizers, a full retrospective recording could be envisioned.

The level of precision (clarity of metadata) in a Digital Library may differ substantially from a "classical library". Mistakes can occur as the gathered metadata is collected by non-librarians and occasionally the syntax is slightly ignored. On the other hand, the speedy and uncomplicated access to ERES-material will enhance the level of attention and therefore make participation in future conferences even more interesting. The searchability of gathered data is to be regarded as an important asset (implementation of a "google-like" interface). For complex retrievals, however, the use of the advanced search feature is advised (Fig. 4).

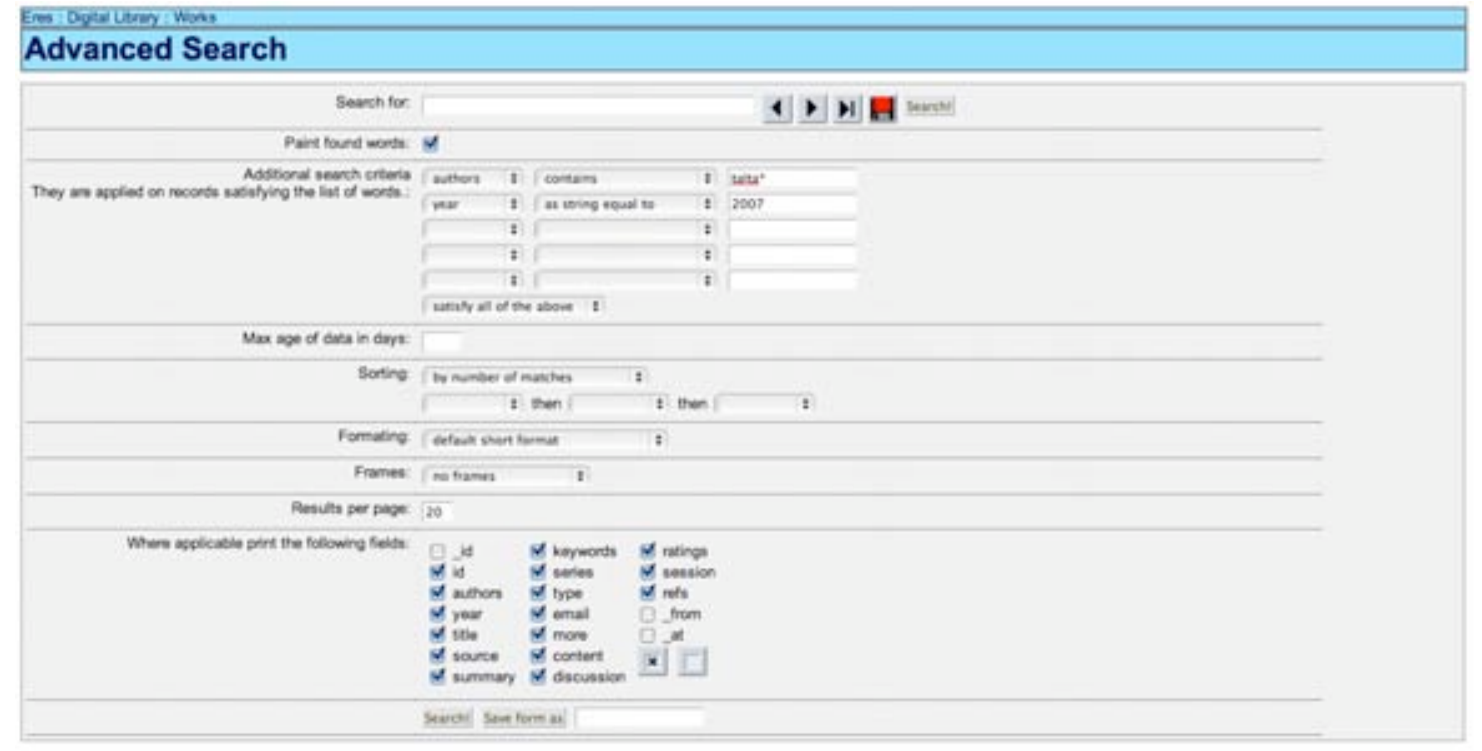

Fig. 4 The advanced search features 


\section{Outlook}

There is no doubt that part of the importance of a conference is to provide a setting where participants can enjoy fruitful exchanges. A Digital Library would, however, allow for a comprehensive overview beyond the scope of a single meeting and enable further exchanges "in between" the conferences. It is crucial for the success of eres.scix.net to assure handling and maintenance on a shoestring budget. The additional workload for conference chairs ought to be manageable, and the pay-off for their contribution is enhanced visibility. To ensure sustainable achievement, forthcoming conferences should be added periodically.

ERES-members are able to "use" the recorded entries on their personal homepages. The author presented in Fig. 2 could, for instance, use the following static link:

\section{http://eres.scix.net/cgi-bin/works/Search?search=authors\%3Ataltavull}

or if needed, also the link of an individual entry as presented in Fig. 3:

\section{http://eres.scix.net/cgi-bin/works/Show? id=eres2006 311}

This could especially be of use for the conference programme and allow to (pre-) publish the papers shortly before the conference.

In this context, the harvesting of metadata defines a possible further "use" of eres.scix.net. Also applications related to the Semantic Web are gaining interest as the number of entries is growing substantially.

\section{References}

[1] Martens, B.; Turk, Z.; Bjoerk, B.-C.; Cooper, Z. "Re-engineering the Scientific Knowledge Management Process: The SciX Project". In: Automation in Construction, 12(2003)6, pp. 677-687.

[2] Alastair A.; Brown, L., "ERES and the Development of Real Estate Research in Europe The First Decade". Powerpoint presentation available at www.eres.org [accessed May 6th 2009]. 